\title{
The impact of pericardial approach and myocardial protection onto postoperative right ventricle function reduction
}

\author{
Marco Zanobini ${ }^{1}$, Claudia Loardi ${ }^{1}$, Paolo Poggio ${ }^{1}$, Gloria Tamborini ${ }^{1}$, Fabrizio Veglia', Alessandro Di Minno ${ }^{1}$, \\ Veronika Myasoedova ${ }^{1}$, Liborio Francesco Mammana ${ }^{1}$, Raoul Biondi ${ }^{1}$, Mauro Pepi ${ }^{1}$, Francesco Alamanni ${ }^{1}$ \\ and Matteo Saccocci ${ }^{1,2^{*}}$
}

\begin{abstract}
Background: The reduction of RV function after cardiac surgery is a well-known phenomenon. It could persist up-to one year after the operation and often leads to an incomplete recovery at follow-up echocardiographic control. The aim of the present study is to analyze the impact of different modalities of pericardial incision (lateral versus anterior) and of myocardial protection protocols (Buckberg versus Custodiol) onto postoperative RV dynamic by relating two- and three-dimensional echocardiographic parameters in patients undergoing mitral valve repair through minimally invasive or traditional surgery approach.

Methods: We have analyzed 44 consecutive patients with severe degenerative mitral regurgitation who underwent mitral reparation with different surgical approach and cardioplegia type: Group 1 (17 pts): sternotomy with Buckberg cardioplegia protocol; Group 2 (10 pts): sternotomy with Custodiol cardioplegia; Group 3 (17 pts): mini-invasive surgery with Custodiol cardioplegia. Two-dimensional transthoracic echocardiography was performed pre- and 6 months post-surgery to evaluate RV function by tricuspid annular plane systolic excursion (TAPSE).

Results: All patients underwent successful and uneventful. A postoperative TAPSE reduction was found in all groups. However, mini-invasive patients experienced a significant reduced variation versus traditional surgery.

Conclusions: Mini-invasive mitral repair, with lateral incision of pericardium, reduces postoperative TAPSE fall, while cardioplegia protocol fails to have an impact onto longitudinal RV function. In our study, the RV seems to experience a clinically irrelevant geometrical modification too, whose entity appears to be less evident in case of lateral pericardial approach. These results could strengthen the use of minimally invasive approach also to preserve RV function.
\end{abstract}

Keywords: Mitral valve, Valve repair, Minimally invasive surgery, Right ventricle, Cardioplegia, Echocardiography

\section{Background}

Right ventricular function is widely known as a determinant of exercise capacity and as significant prognostic value in the evaluation of surgical outcome $[1,2]$. During and immediately after cardiac surgery, it is known that there is a decrease of two-dimensional indexes of right ventricle systolic performance [3-5]. Recovery to

\footnotetext{
* Correspondence: matteo.saccocci@unimi.it

'Department of Cardiac Surgery, Centro Cardiologico Monzino IRCCS,

University of Milan, Via Parea, 4, 20138 Milan, Italy

${ }^{2}$ Heart Center, University Hospital of Zürich, University of Zürich, Zürich, $\mathrm{CH}$ Switzerland
}

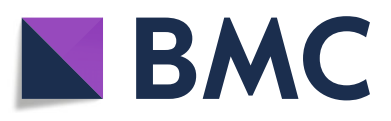

(c) The Author(s). 2018 Open Access This article is distributed under the terms of the Creative Commons Attribution 4.0 International License (http://creativecommons.org/licenses/by/4.0/), which permits unrestricted use, distribution, and reproduction in any medium, provided you give appropriate credit to the original author(s) and the source, provide a link to the Creative Commons license, and indicate if changes were made. The Creative Commons Public Domain Dedication waiver (http://creativecommons.org/publicdomain/zero/1.0/) applies to the data made available in this article, unless otherwise stated.

basal values is often incomplete and an echocardiographic dysfunction can persist even at one year after surgery [6].

Physiopathology of right ventricle behavior following cardiac surgery is a largely debated issue and several hypotheses have been suggested: 1) myocardial hypothermia [7]; 2) cardiopulmonary bypass use [8]; 3) pericardial adhesions [9]. Pericardial opening need [10] and modality of cardioplegia delivery, retrograde cardiac protection seems to be less effective in preserving right ventricle function, [11] appear to be mainly involved.

Two-dimensional echocardiography represents the reference method for cardiac surgery patients' follow up; 
however, regarding the evaluation of right ventricle performance, it has important limitations due to its particular anatomy. The recent introduction of 3-dimensional echocardiographic images allows a more complete evaluation of right ventricle contraction, showing that two-dimensional indexes decrease failed to be accompanied by concomitant parallel right ventricle three-dimensional functional changes [3].

Unfortunately, 3D echocardiograms can be performed only by highly specialized experienced operators. Indeed, 2D measurements like tricuspid annular plane systolic excursion (TAPSE) is still largely used in right ventricular evaluation.

The aim of the present study is to evaluate the impact of the type of pericardial incision (lateral versus anterior) in combination of different myocardial protection protocols onto postoperative right ventricular systolic function in order to further investigate the superiority of minimally invasive approach in mitral valve surgery.

\section{Methods}

Population and study protocol

All patients were enrolled in our Center and operated by the same surgeon. Written informed consent to participate in this observational study, which was approved by Centro Cardiologico Monzino Institutional Review Board, was obtained from all patients. The study protocol conforms to the ethical guidelines of the Declaration of Helsinki as reflected in a priori approval by the institution's human research committee.

To achieve the aim of this study, we compared 2- and 3-dimensional echocardiographic parameters in patients undergoing minimally invasive or traditional (full sternotomy) mitral valve repair (MVR) focusing onto the impact of pericardial incision and cardioplegia protocol.
We retrospectively analyze data of 44 consecutive patients (mean age $54 \pm 12$ years; 34 males/10 females) with severe mitral regurgitation related to degenerative dysfunction due to mitral valve prolapse who underwent mitral valve reparation at our Center by the same Surgeon. We subdivided them in 3 groups according to surgical approach and cardioplegia type (Fig. 1):

1. Group 1: traditional sternotomy operation with Buckberg cardioplegia protocol (blood mixed antegrade-retrograde solution) - 17 patients

2. Group 2: traditional sternotomy operation with Custodiol cardioplegia (crystalloid antegrade administration) - 10 patients

3. Group 3: mini-invasive surgery $(4 \mathrm{~cm}$ right anterolateral thoracotomy) with Custodiol cardioplegia (crystalloid antegrade administration) - 17 patients

Mini-invasive mitral surgery tends to be performed with single shot cardioplegia protocol, like Custodiol, while Buckberg protocol seems to be more protective and versatile for traditional surgery. The design of the study permits to investigate independently surgical approach and cardioplegia making a comparison between groups with same pericardial access (group 1 and 2) but different myocardial protection and between groups with same cardioplegia protocol but different incision of the pericardium (group 2 and 3 ).

Clinical and echocardiographic baseline patients' characteristics are shown in Table 1. Composition of cardioplegic solutions is reported in Table 2.

Exclusion criteria were persistent or paroxysmal atrial fibrillation, urgent intervention with hemodynamic instability, poor echocardiographic acoustic apical window, tricuspid regurgitation major than 1 degree (scale 1 to 4 ),

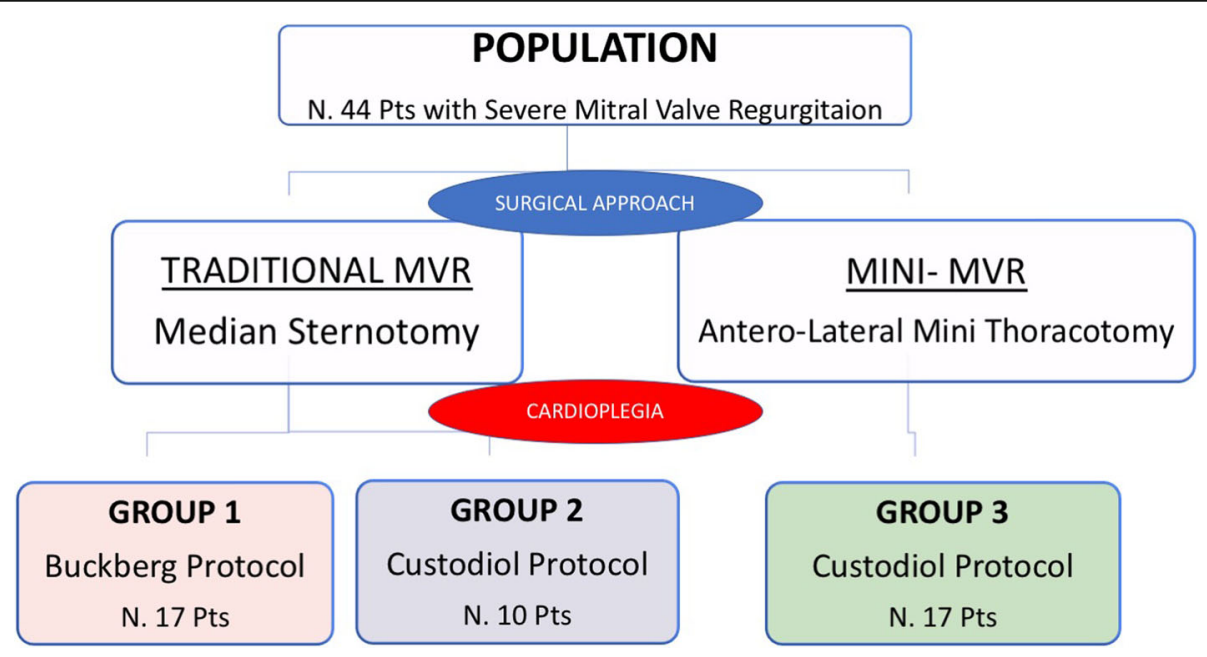

Fig. 1 Design of the study 
Table 1 Clinical and echocardiographic groups' baseline characteristics

\begin{tabular}{lllll}
\hline & $\begin{array}{l}\text { Group 1 } \\
(n=17)\end{array}$ & $\begin{array}{l}\text { Group 2 } \\
(n=10)\end{array}$ & $\begin{array}{l}\text { Group 3 } \\
(n=17)\end{array}$ & P value \\
\hline Age, y & $54.8 \pm 11.9$ & $59.2 \pm 10.2$ & $50.9 \pm 12.2$ & 0.14 \\
Male, $\mathrm{n}(\%)$ & $13(76 \%)$ & $7(70 \%)$ & $14(82 \%)$ & 0.18 \\
BSA $\left(\mathrm{m}^{2}\right)$ & $1.9 \pm 0.2$ & $1.8 \pm 0.2$ & $1.8 \pm 0.1$ & 0.32 \\
EuroSCORE 2 & $0.98 \%$ & $0.96 \%$ & $0.95 \%$ & 0.45 \\
LVEF & $60.4 \pm 6.4$ & $58.2 \pm 4.1$ & $64.5 \pm 4.7$ & 0.32 \\
TAPSE (mm) & $24.5 \pm 4.8$ & $27.4 \pm 5.3$ & $23.5 \pm 4$ & 0.17 \\
SPAP (mmHg) & $33.9 \pm 4.8$ & $32.3 \pm 5.1$ & $34.9 \pm 2.7$ & 0.83 \\
RVEF (3D) & $62.2 \pm 8.9$ & $60.5 \pm 8.2$ & $58.5 \pm 6.1$ & 0.56 \\
RVSV (3D) & $68.3 \pm 23.9$ & $54.1 \pm 18.8$ & $68 \pm 13.1$ & 0.21 \\
RVESV (3D) & $43.4 \pm 22.5$ & $34.7 \pm 11.7$ & $48.5 \pm 11.6$ & $0.34^{\dagger}$ \\
RVEDV (3D) & $111.7 \pm 42.8$ & $88.8 \pm 26.1$ & $116.5 \pm 19.8$ & $0.18^{\varphi}$ \\
$\begin{array}{l}\text { MVP Type } \\
\quad\end{array}$ & & & & \\
$\quad$ Posterior leaflet & 17 & 9 & 17 & \\
$\quad \begin{array}{l}\text { Anterior leaflet } \\
\text { prolapse }\end{array}$ & 2 & 1 & & \\
\hline
\end{tabular}

*EuroSCORE 2 denotes the European System for Cardiac Operative Risk Evaluation (2nd version)

${ }^{+} p=0.04$ Group 2 versus 3

${ }^{\varphi} p=0.03$ Group 2 versus 3

$B S A$ Indicates body surface area, LVEF Left ventricular ejection fraction, TAPSE Tricuspid annular plane systolic excursion, SPAP Systolic pulmonary arterial pressure, RVEF Right ventricular ejection fraction, RVSV Right ventricular stroke volume, RVESV Right ventricular end-systolic volume, RVEDV Right ventricular end-diastolic volume, MVP Mitral valve prolapse

concomitant surgery procedures or mitral valve replacement, major pulmonary diseases justifying a right ventricular dysfunction or pulmonary hypertension, previous cardiac surgery and preoperative reduced LV function (Ejection fraction <40\%). Two-dimensional (2D-) and three-dimensional (3D-) transthoracic echocardiography (TTE) was performed preoperative (24-48 $\mathrm{h}$ before surgery) and 6 months after surgical operation.

Table 2 Composition of cardioplegic solutions used in the study

\begin{tabular}{lllll}
\hline Ingredient & $\begin{array}{l}\text { Buckberg cold } \\
\text { induction 4.1 }\end{array}$ & $\begin{array}{l}\text { Buckberg cold } \\
\text { maintenance 4.1 }\end{array}$ & $\begin{array}{l}\text { Buckberg } \\
\text { "hot shot" }\end{array}$ & Custodiol \\
\hline $\mathrm{Na}^{+}$ & 140 & 140 & 140 & 15 \\
$\mathrm{~K}^{+}$ & 20 & 10 & 8 & 9 \\
$\mathrm{Mg}^{++}$ & 13 & 9 & 6 & 4 \\
$\mathrm{Ca}^{++}$ & - & - & - & 0.015 \\
Hystidine & - & - & - & 198 \\
Tryptophan & - & - & - & 2 \\
Ketoglutarate & - & - & - & 1 \\
Mannitol & - & - & - & 30 \\
Glucose & 6 & 6 & 8 & - \\
pH & 7.2 & 7.4 & 7.5 & $7.02-7.2$ \\
\hline
\end{tabular}

All ingredients are expressed in $\mathrm{mmol} / \mathrm{L}$

\section{Surgical procedures}

Traditional MVR patients (group 1 and 2) underwent complete median sternotomy and anterior opening of the pericardium with a reversed T incision. Standard cardiopulmonary bypass (CBP) was implanted with ascending aortic cannulation and bicaval venous cannulation.

In Group 1 Buckberg cardioplegia was adopted, consisting in a three-phases myocardial protection protocol:

1. Cold induction: delivery of cold cardioplegic solution $\left(8-12{ }^{\circ} \mathrm{C}\right)$ antegrade and retrograde for 2 min each until complete cardioplegic arrest was achieved (flow $200 \mathrm{ml} / \mathrm{min}$, in hypertrophied hearts increase to $300 \mathrm{ml} / \mathrm{min}$ )

2. Reinfusions with cold blood cardioplegia: during aortic cross-clamping, multidose cold blood cardioplegia was applied at intervals of $20 \mathrm{~min}$ to maintain cardioplegic arrest and myocardial hypothermia. Infusions were delivered retrograde for $1 \mathrm{~min}$ (flow $200 \mathrm{ml} / \mathrm{min}$ )

3. Warm terminal reperfusion ("hot shot"): normothermic substrate-enriched blood cardioplegia was applied before aortic unclamping. The warm reperfusate was delivered via coronary sinus for $1 \mathrm{~min}$ and followed by a brief (20-30 s) retrograde administration of normothermic blood [12].

In Group 2 Custodiol protocol protection (single antegrade injection of crystalloid intracellular cold cardioplegia in 6-8 $\mathrm{min}$ ) was employed.

Minimally invasive mitral valve repair (Group 3) was performed through a small $(5 \mathrm{~cm})$ right antero-lateral thoracotomy and with a lateral supra-phrenic pericardial incision. Peripheral CBP was implanted using femoral vessels for arterial and venous cannulation. Similarly to Group 2, a single antegrade dose of Custodiol cardioplegia was administered.

All interventions were performed in moderate hypothermia $\left(32{ }^{\circ} \mathrm{C}\right)$ with a direct left atriotomy through Waterstone's groove. Depending on patient's mitral alteration we used different surgical repairing techniques always supported by annuloplasty with a prosthetic ring implantation (Table 3). In each group the pericardium was completely re-closed with a continuous suture.

\section{Echocardiographic measurements}

We performed a complete standard M-mode and twodimensional echocardiographic examinations using a Philips ultrasound system (iE33, Andover, MA, USA) and an S5-1 sector array probe. LV end-diastolic and end-systolic volumes, as well as biplane ejection fraction were measured in apical four- and two chamber views 
Table 3 Intraoperative groups' characteristics

\begin{tabular}{|c|c|c|c|c|}
\hline & $\begin{array}{l}\text { Group } 1 \\
(n=17)\end{array}$ & $\begin{array}{l}\text { Group } 2 \\
(n=10)\end{array}$ & $\begin{array}{l}\text { Group 3 } \\
(n=17)\end{array}$ & $P$ value \\
\hline CPB time (min) & $113 \pm 17$ & $105 \pm 18$ & $131 \pm 23$ & 0.21 \\
\hline Cross-clamp time (min) & $95 \pm 13$ & $93 \pm 12$ & $112 \pm 12$ & 0.24 \\
\hline $\begin{array}{l}\text { Complete prosthetic } \\
\text { semi-rigid ring }\end{array}$ & 3 & 2 & 2 & 0.32 \\
\hline Incomplete band & 14 & 8 & 15 & 0.25 \\
\hline Annular plication & 1 & 0 & 0 & 0.43 \\
\hline Quadrangular resection & 6 & 4 & 7 & 0.44 \\
\hline Triangular resection & 9 & 5 & 9 & 0.28 \\
\hline Sliding-plasty & 5 & 4 & 6 & 0.12 \\
\hline $\begin{array}{l}\text { Artificial chordae } \\
\text { positioning }\end{array}$ & 2 & 1 & 1 & 0.23 \\
\hline $\begin{array}{l}\text { Papillary muscle } \\
\text { plication }\end{array}$ & 1 & 0 & 0 & 0.34 \\
\hline
\end{tabular}

$C P B$ Indicates cardio-pulmonary bypass

with the area-length method. Systolic pulmonary arterial pressure was non-invasively acquired using Doppler echo method from the systolic right ventricle-right atrial gradient, calculated from the systolic trans-tricuspid regurgitant flow peak velocity by the modified Bernoulli equation. Right atrial (RA) pressure was derived by means of the inferior vena cava (IVC) collapsibility index measured from the subcostal view [13]. To estimate tricuspid annular plane systolic excursion (TAPSE), defined as the difference in the displacement of the right ventricle base from end-diastole to end-systole, from the apical four-chamber view, the M-mode cursor was positioned at the junction of the tricuspid valvular plane with the right ventricle free $[14,15]$.

3D- Real-time TTE was performed during same echocardiographic session utilizing an $\mathrm{X} 3-1$ matrix array probe. The 3D-measurement were acquired using 'full volume' mode from the apical view, adapted to improve the visualization of the right ventricular chamber. We registered Two datasets for each patient. To perform offline post-processing and three-dimensional reconstruction we used a commercially available dedicated system (Echo View, Tom Tec Imaging Inc., Munich, Germany) equipped with a four-dimensional right ventricle analysis software [16].

\section{Statistical analysis}

Data were managed in Microsoft Excel 2016 and analyzed with SPSS 22.0 software (SPSS, Inc., Chicago, IL) and SAS system (SAS Institute Inc., Cary, NC). Each echocardiographic parameter was evaluated pre- and 6 months post-surgery. Continuous variables were presented as mean $\pm \mathrm{SD}$ and compared with an unpaired $t$ test, while categorical data were expressed as percentages or numbers and compared with $K^{2}$ test.
A between-groups comparison examining the impact of pericardial approach and type of cardioplegia on right ventricular function over time was made with an analysis of variance and co-variance (ANOVA test) adjusted for patients' age, sex, body surface area and right ventricular features basal values.

A $p$ value $<0.05$ was considered as statistically significant.

\section{Results}

All enrolled patients underwent mitral valve repair surgery without significant complications. After six month after surgery (185 \pm 23 days), all cases had residual mitral regurgitation inferior to 1 degree (scale 1 to 4 ). At least one good quality, three-dimensional right ventricle dataset was acquired in all patients before surgery and six months after the surgery. Tables 4 and 5 and Panel A (Fig. 2) shows the mean values of the two-dimensional and three-dimensional parameters for each step of the study in both groups.

Table 4 Two-dimensional and three-dimensional echocardiographic parameters measured at pre- and 6 months post-surgery

\begin{tabular}{|c|c|c|c|}
\hline Variable & Pre-surgery & Sixth month & P Within Group \\
\hline \multicolumn{4}{|l|}{ TAPSE (mm) } \\
\hline Group 1 & $24.5 \pm 4.8$ & $14.9 \pm 3$ & $<0.0001$ \\
\hline Group 2 & $27.4 \pm 5.3$ & $19.3 \pm 4.2$ & 0.0003 \\
\hline Group 3 & $23.5 \pm 4$ & $21.5 \pm 4.1$ & 0.013 \\
\hline \multicolumn{4}{|c|}{ SPAP (mmHg) } \\
\hline Group 1 & $33.9 \pm 4.8$ & $25.7 \pm 2.9$ & $<0.0001$ \\
\hline Group 2 & $32.3 \pm 5.1$ & $28.3 \pm 6.5$ & 0.054 \\
\hline Group 3 & $34.9 \pm 8.1$ & $25.2 \pm 3.1$ & 0.049 \\
\hline \multicolumn{4}{|l|}{ RVEDV (ml) } \\
\hline Group 1 & $111.7 \pm 42.8$ & $93.1 \pm 26.2$ & 0.016 \\
\hline Group 2 & $88.8 \pm 26.1$ & $72.2 \pm 21.3$ & 0.002 \\
\hline Group 3 & $116.5 \pm 19.8$ & $105.2 \pm 20.6$ & 0.039 \\
\hline \multicolumn{4}{|l|}{ RVESV (ml) } \\
\hline Group 1 & $43.4 \pm 22.5$ & $39.2 \pm 13.6$ & 0.244 \\
\hline Group 2 & $34.7 \pm 11.7$ & $29.2 \pm 9.2$ & 0.048 \\
\hline Group 3 & $48.5 \pm 11.6$ & $41 \pm 9$ & 0.005 \\
\hline \multicolumn{4}{|l|}{ 3D RVEF (\%) } \\
\hline Group 1 & $62.2 \pm 8.9$ & $58.2 \pm 5.4$ & 0.021 \\
\hline Group 2 & $60.5 \pm 8.2$ & $59.5 \pm 6.9$ & 0.579 \\
\hline Group 3 & $58.5 \pm 6.1$ & $60.7 \pm 6.8$ & 0.207 \\
\hline \multicolumn{4}{|c|}{ 3D RVSV (ml) } \\
\hline Group 1 & $68.3 \pm 23.9$ & $53.9 \pm 13.9$ & 0.004 \\
\hline Group 2 & $54.1 \pm 18.8$ & $43 \pm 14.1$ & 0.0039 \\
\hline Group 3 & $68 \pm 13.1$ & $64.1 \pm 15.9$ & 0.349 \\
\hline
\end{tabular}

TAPSE Indicates tricuspid annular plane systolic excursion, SPAP Systolic pulmonary arterial pressure, RVEDV Right ventricular end-diastolic volume, RVESV Right ventricular end-systolic volume, RVEF Right ventricular ejection fraction, RVSV Right ventricular stroke volume 
Table 5 Inter-group comparison of two-dimensional and three-dimensional echocardiographic parameters variations (6 months post-surgery versus pre-operative values)

\begin{tabular}{lllllll}
\hline Delta variable & Group 1 & Group 2 & Group 3 & P Group 1 vs 3 & P Group 1 vs 2 & $P$ Group 3 vs 2 \\
\hline TAPSE $(\mathrm{mm})$ & -9.5 & -8.1 & -2 & $<0.0001$ & 0.12 & 0.008 \\
SPAP $(\mathrm{mmH})$ & -8.1 & -4 & -9.7 & 0.66 & 0.86 & 0.65 \\
RVEDV $(\mathrm{ml})$ & -18.6 & -16.5 & -11.3 & 0.54 & 0.93 & 0.51 \\
RVESV $(\mathrm{ml})$ & -4.2 & -5.5 & -7.4 & 0.49 & 0.69 & 0.91 \\
3D RVEF $(\%)$ & -3.9 & -0.9 & 2.2 & 0.04 & 0.22 & 0.74 \\
3D RVSV $(\mathrm{ml})$ & -14.4 & -11.1 & -3.9 & 0.18 & 0.66 & 0.45 \\
2D LVEF (\%) & -7.6 & -8.1 & -3.8 & 0.08 & 0.84 & 0.19 \\
\hline
\end{tabular}

TAPSE indicates tricuspid annular plane systolic excursion, SPAP systolic pulmonary arterial pressure, RVEDV right ventricular end-diastolic volume, RVESV right ventricular end-systolic volume, RVEF right ventricular ejection fraction, RVSV right ventricular stroke volume, LVEF left ventricular ejection fraction

Preoperative left and right ventricular function were in normal range for all patients. Basal TAPSE was slightly greater, but did not reach statistical significance, in traditional surgery (Group 1 and 2, respectively 24.5 and 27. 4 versus 23.5 for mini-invasive patients; $p=0.17$ ). All the three groups had similar preoperative 3D right ventricular function and cross-clamping/extracorporeal circulation time.

\section{Two-dimensional measurements}

Postoperative TAPSE fall was found in each group (Table 4), but mini-invasive patients experienced a less marked variation (group 3: $21.5 \pm 4.1$ post- versus $23.5 \pm 4$ pre-; $p=0.01)$ compared to traditional surgery (Group $114.9 \pm 3$ post versus $24.5 \pm 4.8$ pre; $p<0.0001$; Group $219.3 \pm 4.2$ post versus $27.4 \pm 5.3$ pre; $p=0.0003$ ). The difference remained statistically significant after adjustment for patients' age, sex, body surface area and basal TAPSE (Group 3 versus $1 p<$ 0.0001 and Group 3 versus $2 p=0.008$ ).

Systolic pulmonary arterial pressure showed a similar postoperative fall in each group.

Left ventricular volumes and ejection fraction decreased after surgery in a similar manner in all patients.

\section{Three-dimensional measurements}

In Sterno-Buckberg patients (Group 1) end-systolic sizedecreasing trend failed to reach statistical significance. However, in the other two groups (groups 2 and 3, Custodiol), both end-diastolic and end-systolic right ventricular volumes significantly diminished after surgery. Any inter-group size changes comparison showed a significant difference (Table 4).

In mini-invasive patients (group 3), right ventricular ejection fraction slightly augmented after surgery $(60.7 \pm$ 6.8 post versus $58.5 \pm 6.1$ pre; $p=0.2$ ), while, in Group 1 decreased $(58.2 \pm 5.4$ post versus $62.2 \pm 8.9$ pre; $p=0$. $02)$. In contrast, no significance difference was found in Group $2(59.5 \pm 6.9$ post versus $60.5 \pm 8.2$ pre; $p=0.58)$. In addition, this variation was significantly different between mini-invasive versus sternotomy-Buckberg patients $(p=0.04)$. Similarly, right ventricular stroke volume diminished after surgery in all patients without significant inter-group differences.

\section{Discussion}

The importance of RV function as an important physiopathology element in many different cardiovascular disease is a well-known phenomenon and it is an already validated prognostic index after cardiac surgery. In the past, obtaining an accurate postoperative evaluation of RV function has been made difficult by its complex anatomy and morphology. This problem was solved by the assessment of tricuspid annulus movement with 2DEchocardiographic analysis that has been proved to be accurate, feasible, simple and reproducible in both normal and pathological patients [17]. The introduction of 3D-Echocardiography has permitted a big step forward in the evaluation of RV volume and function throughout the cardiac cycle $[18,19]$ permitting to calculate the right ventricular ejection fraction (RV-EF) that represents the global performance of the ventricle. The main limitation 3Dechocardiography is that is a technology usually available only in high-experienced center with skilled operators while 2D- TAPSE evaluation is an easy measurement for every echocardiographist.

Several studies report a reduction of TAPSE after cardiac surgery for congenital and acquired diseases $[6,20]$ without univocal explanation. Observing a postoperative TAPSE decrease, without variation in left ventricle function, has driven to interpret it as a simple modification with modest clinical impact. Many hypothesis have been presented in order to clarify this loss in RV performance measured at 2D-TTE, including cardiopulmonary bypass use $[8,21]$, geometrical changes of the right ventricular chamber (in association with interventricular septal paradoxical motion [22], intraoperative ischemia, right atrial injury due to cannulation procedure [23], poor myocardial protection [24], and 


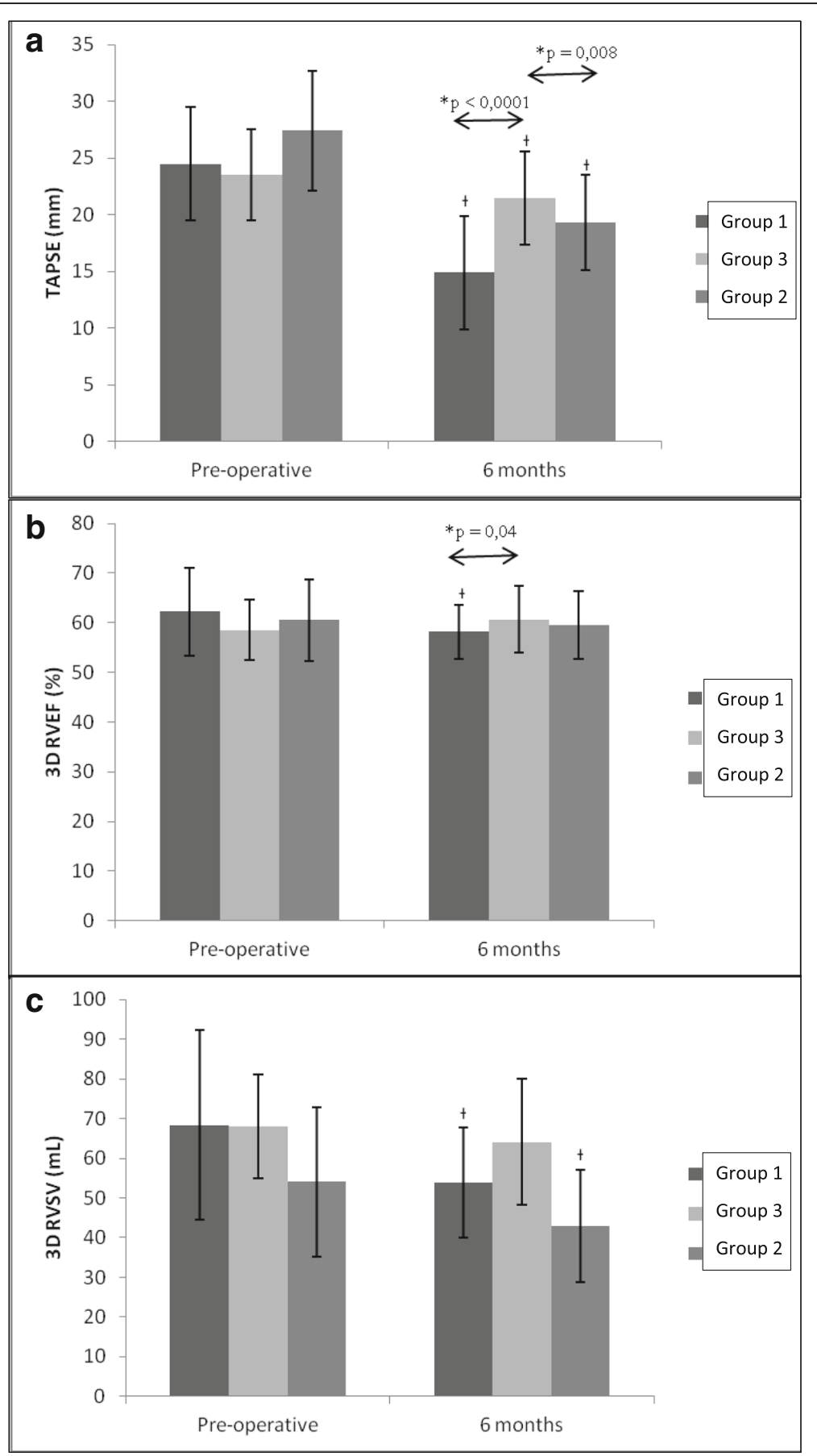

Fig. 2 PANEL a - Mean tricuspid annular plane systolic excursion (TAPSE) and 95\% confidence intervals (CIs) measured preoperatively and at 6 months postoperatively. ${ }^{*}$ Between-groups comparison. ${ }^{\dagger} p<0.05$ vs preoperative; PANEL $\mathbf{b}$ - Mean three-dimensional right ventricular ejection fraction (3D RVEF) and 95\% confidence intervals (Cls) measured preoperatively and at 6 months postoperatively. * Between-groups comparison ${ }^{+} p<0.05$ vs preoperative; Panel $\mathbf{c}$ - Mean three-dimensional right ventricular stroke volume (3D RVSV) and 95\% confidence intervals (CIs) measured preoperatively and at 6 months postoperatively. ${ }^{+} p<0.05$ vs preoperative

extra myocardial causes (pericardial disruption, changes in Fossa Ovalis and post-operative adherence of the right ventricle to the thoracic wall, 9). The role of pericardial injury has also been highlighted in our precedent study [25]. One of the major confounding factors comparing traditional and mini-invasive surgery is represented by the use of single shot cardioplegia protocol in miniMVR. To eliminate this bias and to evaluate myocardial protection impact on TAPSE we designed this three groups study. 
To minimize all other possible confounding factors and to eliminate inter-operators difference, we acquired data only from patient underwent MVR by the same surgeon and who had pre- and postoperative TTE by one dedicated echocardiographist. Surgical valve repair technique doesn't significantly differ within the 3 groups (Table 3) as well as the type of implanted prosthetic rings. Cross-clamping time and cardiopulmonary bypass time resulted comparable in all groups. The pericardial opening was entirely closed with a running suture in all patients.

TAPSE reduction was observed in all groups, but it has been significantly less marked in mini-invasive surgery group who underwent mini-anterolateral thoracotomy with supraphrenic lateral pericardial incision and Custodiol cardioplegia. Evaluating different cardioplegia protocol with same surgical approach (group 1 vs. 2) we did not find any significant differences. 3Dechocardiographic RV postoperative volume resulted comparable within the 3 groups. A slightly augmented RV ejection fraction was observed after mini-invasive surgery (group 3) while it decreased in patients underwent traditional median sternotomy despite the different cardioplegia protocol (group 1 Buckberg, group 2 Custodiol). According to these results, only different surgical approach impact RV function, in particular, lateral pericardial incision used in mini-invasive surgery is able to significantly limit right-ventricle longitudinal postoperative function decrease while the different type of cardioplegia protocol has no relevant effect on TAPSE.

To explain these findings more than a hypothesis can be provided. The first one could be that anterior pericardial incision modifies a portion of pericardium directly connected with the RV free wall while lateral opening, in the face of interatrial groove, does not interfere with RV motility. As we previously proposed [25], another explication could be linked to the shape itself of anterior pericardial incision. It consisted in a reversed $\mathrm{T}$ incision with a double opening line along the diaphragm that could modify the relationship between this muscle and the inferior RV wall leading to possible variations in longitudinal right ventricular contractile pattern and consequently to postoperative TAPSE fall. The different surgical repair techniques and prosthetic rings used to restore mitral valve competency and to assure long-term durability should not influence inter-group differences due to the homogenous distribution of them in the 3 groups. Different kind of resection or ring did not reach significant statistic difference within the study population (Table 3).

An additional important observation from this study is that, in parallel to the attended impairment of right ventricle long-axis function, also three-dimensional global systolic indexes showed a postoperative slight decreasing trend, which was less accentuated (or even inversed when talking about right ventricular ejection fraction) in miniinvasive group. In other words, it seems that, in our study, the right ventricle undergoes a kind of geometrical modifications too, which appear to be less affected by a lateral pericardial approach. Nevertheless, in our opinion, such tendency (which could be interpreted as clinically negligible since included in the normal range of measure variability) is partially different from what previously reported [3]. A prudent interpretation is justified by various considerations: 1 ) the increasing trend of right ventricular ejection fraction in mini-invasive group is not confirmed by a parallel stroke volume time-course; 2) the inter-group evaluation failed to show a statistically significant difference when comparing Group 3 vs Group 2 (thus perhaps suggesting a role played by cardioplegia too); 3 ) the intragroup three-dimensional variables variations were not significant in all groups.

Basing on these reasons, we believe that deeper investigations involving a greater patients' population and a longer follow up time (which could elucidate that such trend was only a temporary phenomenon, as also pointed out by postoperative right ventricular ejection fraction fluctuations described by Tamborini et al.,3) are required before drawing definitive conclusions.

We studied a relative small number of cases. Despite this limitation and even though our data should be confirmed in a larger population, statistical analysis clearly defined significant changes and differences in the three groups, being able to highlight how different surgical protocols resulted in diverse right ventricular function postoperative trends.

\section{Study limitations}

Main study limitation is represented by limited number of patients of the cohort analyzed and as well as the needed of longer echocardiographic follow-up. Larger population, different type of cardioplegia (Del Nido, St. Thomas, etc..) should be investigated.

\section{Conclusions}

Minimally invasive mitral repair with lateral pericardial opening reduces postoperative TAPSE fall while cardioplegia protocol fails to have an impact onto longitudinal RV function. In our study, the right ventricle seems to show a clinically irrelevant geometrical modification too, whose entity appears to be less evident in case of lateral pericardial approach. These results could strengthen the use of minimally invasive approach also to preserve right ventricle function.

\section{Abbreviations}

CBP: Cardiopulmonary bypass; IVC: Inferior vena cava; MVR: Mitral valve repair; TAPSE: Tricuspid annular plane systolic excursion; TTE: Transthoracic echocardiography 


\section{Authors' contribution}

MS, MZ have contribute equally to this work. MS,CL,MZ,GT, MP data collection, study design. FV,MS, MZ statistical analysis. MS,MZ,CL,PP,ADM,VM,RB writing. MS,MZ,FA,MP,GT manuscript revision. All authors read and approved the final manuscript.

\section{Availability of data and materials}

Available from corresponding author on reasonable request.

\section{Ethics approval and consent to participate}

Written informed consent to participate in this observational study, which was approved by Centro Cardiologico Monzino Institutional Review Board, was obtained from all patients. The study protocol conforms to the ethical guidelines of the Declaration of Helsinki as reflected in a priori approval by the institution's human research committee.

\section{Competing interests}

None of the authors have competing interest within this paper.

\section{Publisher's Note}

Springer Nature remains neutral with regard to jurisdictional claims in published maps and institutional affiliations.

Received: 8 October 2017 Accepted: 9 May 2018

Published online: 05 June 2018

\section{References}

1. De Groote P, Millaire A, Foucher-Hossein C, et al. Right ventricular ejection fraction is an independent predictor of survival in patients with moderate heart failure. J Am Coll Cardiol. 1998;32:948-54.

2. Davila-Roman VG, Waggoner AD, Hopkins WE, Barzilai B. Right ventricular dysfunction in low output syndrome after cardiac operations: assessment by transesophageal echocardiography. Ann Thorac Surg. 1995;60:1081-6.

3. Tamborini $G$, Muratori $M$, Brusoni $D$, et al. Is right ventricular systolic function reduced after cardiac surgery? A two- and three- dimensional echocardiographic study. Eur J Echocardiogr. 2009;10:630-4.

4. Unsworth B, Casula R, Kyriacou A, et al. The right ventricular annular velocity reduction caused by coronary artery bypass graft surgery occurs at the moment of pericardial incision. Am Heart J. 2010;159:314-22.

5. Wranne B, Pinto FJ, Hammarström E, St Goar FG, Puryear J, Popp RL. Abnormal right heart filling after cardiac surgery: time course and mechanisms. Br Heart J. 1991;66:435-42.

6. Alam M, Hedman A, Nordlander R, Samad B. Right ventricular function before and after an uncomplicated coronary artery bypass graft as assessed by pulsed wave Doppler tissue imaging of the tricuspid annulus. Am Heart J. 2003:146:520-6.

7. Boldt J, Kling D, Dapper F, Hempelmann G. Myocardial temperature during cardiac operations: influence on right ventricular function. J Thorac Cardiovasc Surg. 1990;100:562-8.

8. Pegg T, Selvanayagam J, Karamitsos T, et al. Effects of off-pump versus onpump coronary artery bypass grafting on early and late right ventricular function. Circulation. 2008;117:2202-10.

9. Joshi S, Salah A, Mendoza D, Goldstein SA, Fuisz AR, Lindsay J. Mechanism of paradoxical ventricular septal motion after coronary bypass grafting. Am J Cardiol. 2009:103:212-5.

10. Unsworth B, Casula R, Yadav H, et al. Contrasting effect of different operations on echocardiographic right ventricular long axis velocities, and implications for interpretation of post-operative values. Int J Cardiol. 2013; 165:151-60.

11. Rangaraj A, Ghanta R, Umakanthan R, et al. Real-time visualization and quantification of retrograde cardioplegia delivery using near infrared fluorescent imaging. J Card Surg. 2008;23:701-8.

12. Rosenkranz ER, Okamoto F, Buckberg GD, et al. Safety of prolonged aortic clamping with blood cardioplegia. III. Aspartate enrichment of glutamateblood cardioplegia in energy-depleted hearts after ischemic and reperfusion injury. J Thorac Cardiovasc Surg. 1986;91:428-35.

13. Pepi M, Tamborini G, Galli C, et al. A new formula for echo-Doppler estimation of right ventricular systolic pressure. J Am Soc Echocardiogr. 1994;7:20-6

14. Hammarstrom E, Wranne B, Pinto FJ, Puryear J, Popp RL. Tricuspid annular motion. J Am Soc Echocardiogr. 1991;4:131-9.
15. Miller D, Farah MG, Keith F, Fox K, Schluchter M, Hoit BD. The relation between quantitative right ventricular ejection fraction andindices of tricuspidal annular motion and myocardial performance. J Am Soc Echocardiogr. 2004;17:443-7.

16. Niemann PS, Pinho L, Balbach T, et al. Anatomically oriented right ventricular volume measurements with dynamic three-dimensional echocardiography validated by 3-tesla magnetic resonance imaging. J Am Coll Cardiol. 2007:50:1668-76.

17. Tamborini G, Pepi M, Galli C, et al. Feasibility and accuracy of a routine echocardiographic assessment of right ventricular function. Int J Cardiol. 2007;115:86-9.

18. Tamborini G, Brusoni D, Torres Molinab JE, et al. Feasibility of a new generation three-dimensional echocardiography for right ventricular volumetric and functional measurements. Am J Cardiol. 2008;102:499-505.

19. Maffessanti F, Muraru D, Esposito R, et al. Age-, body size-, and sex-specific reference values for right ventricular volumes and ejection fraction by three-dimensional echocardiography: a multicenter echocardiographic study in 507 healthy volunteers. Circ Cardiovasc Imaging. 2013;6:700-10.

20. Hanseus KC, Bjorkhem GE, Brodin LA, Pesonen E. Analysis of atrioventricular plane movements by Doppler tissue imaging and $\mathrm{M}$-mode in children with atrial septal defects before and after surgical and device closure. Pediatr Cardiol. 2002:23:152-9.

21. Forsberg L, Tamas E, Vanky F, Nielsen NE, Enqvall J, Nylander E. Left and right ventricular function in aortic stenosis patients 8 weeks posttranscatheter aortic valve implantation or surgical aortic valve replacement. Eur J Echocardiogr. 2011;12:603-11.

22. Roshanali F, Yousefnia M, Mandegar M, Rayatzadeh H, Alinejad S. Decreased right ventricular function and coronary artery bypass grafting. Tex Heart Inst J. 2008;35:250-5

23. Lindqvist $P$, Holmgren A, Zhao J, Henein MY. Effect of pericardial repair after aortic valve replacement on septal and right ventricular function. Int J Cardiol. 2012;155:388-93.

24. Jasinski M, Kadziola Z, Bachowski R, et al. Comparison of retrograde versus anterograde cold blood cardioplegia: randomized trial in elective coronary artery bypass patients. Eur J Cardiothorac Surg. 1997:12:620-6.

25. Zanobini M, Saccocci M, Tamborini G, et al. Postoperative echocardiographic reduction of right ventricular function: is pericardial opening modality the main culprit? Biomed Res Int. 2017;2017:4808757. https://doi.org/10.1155/2017/4808757.

\section{Ready to submit your research? Choose BMC and benefit from:}

- fast, convenient online submission

- thorough peer review by experienced researchers in your field

- rapid publication on acceptance

- support for research data, including large and complex data types

- gold Open Access which fosters wider collaboration and increased citations

- maximum visibility for your research: over $100 \mathrm{M}$ website views per year

At BMC, research is always in progress.

Learn more biomedcentral.com/submissions 